\title{
LiDAR as a Valuable Information Source for Habitat Mapping
}

\author{
Sander MÜCHER ${ }^{1}$, Laure ROUPIOZ ${ }^{1}$, Henk KRAMER ${ }^{1}$, Michel WOLTERS ${ }^{1}$, \\ Marion BOGERS ${ }^{1}$, Richard LUCAS ${ }^{2}$, Peter BUNTING ${ }^{2}$, Zisis PETROU $^{3}$, \\ Vasiliki KOSMIDOU ${ }^{3}$, Ioannis MANAKOS ${ }^{3}$, Emilio PADOA-SCHIOPPA ${ }^{4}$, \\ Gentile Francesco FICETOLA ${ }^{4}$, Anna BONARDI ${ }^{4}$, Maria ADAMO $^{5}$ and Palma BLONDA ${ }^{5}$ \\ ${ }^{1}$ ALTERRA, Wageningen/Netherlands·sander.mucher@wur.nl \\ ${ }^{2}$ Institute of Geography and Earth Sciences, Aberystwyth University/UK \\ ${ }^{3}$ Centre for Research \& Technology Hellas, Thessaloniki/Greece \\ ${ }^{4}$ Università degli Studi di Milano Bicocca, Milano/Italy \\ ${ }^{5}$ CNR-ISSIA, Bari/Italy
}

This contribution was double-blind reviewed as extended abstract.

\begin{abstract}
Key is the challenge to develop a biodiversity observation system that is transmissible and cost effective. Measuring and reliable reporting of trends and changes in biodiversity requires that data and indicators are collected and analysed in a standard and comparable way. LiDAR is an alternative remote sensing technology that allows to increase the accuracy of biophysical measurements and to extend spatial analysis into the third dimension. The BIO_SOS project shows alternatives to measure habitat diversity as a proxy for biodiversity on the basis of plant life forms. The objective of our study is to assess to what extent LiDAR can be used to map and monitor plant life forms and associated General Habitat Categories (GHCs). The conclusions are that LiDAR provides accurate height measurements on shrubs and trees, even in early spring when no leaves are present. Canopy height models as derived from LiDAR and in combination with very high resolution satellite imagery provides a powerful tool with for the identification of plant life forms and as a direct input for spatial modelling of species distribution. Since LiDAR data are not everywhere available, finding alternatives for height feature extraction from optical imagery for might be worthwhile.
\end{abstract}

\section{Introduction}

LiDAR (LIght Detection And Ranging or Laser Imaging Detection And Ranging) is an active remote sensing technique that measures the properties of emitted scattered light to determine the 3D coordinates $(\mathrm{x}, \mathrm{y}, \mathrm{z})$ and other properties of a distant target (ST-ONGE 2005). To do so, the LiDAR instrument transmits light pulses out to a target and calculates the distance based on light. The time for light pulses to return back to the LiDAR sensor is used to calculate the distance to the target. The "xy" accuracy of the pulse center is typically $0.1-0.5 \mathrm{~m}$. The accuracy in " $\mathrm{z}$ " is usually better than $0.2 \mathrm{~m}$. Thus, LiDAR, in con- 
trast to optical remote sensing techniques, can be expected to bridge the gap in structural information at the landscape scale. LiDAR was originally introduced to generate more accurate Digital Elevation Models (DEMs), but has recently become an effective tool for natural resources application. Recent studies have explored LiDAR as a new source of geospatial data that can provide fine-grained information about the 3-D physical structure of terrestrial and aquatic ecosystems (GEERLING et al. 2007). LiDAR remote sensing shows considerable efficacy for habitat mapping in fine detail across broad areas. It may replace many labour-intensive, field-based measurements, and can characterize habitat in novel ways. Considering monitoring applications, the repeatable and high absolute "xyz" accuracy is advantageous since changes can be detected at sub-meter scales and the same measurement units can be monitored over time. In that sense, LiDAR constitutes an efficient tool for short and long term monitoring of changes in surface structure and vegetation. In regards to this perspective the BIO_SOS project considered using LiDAR as a valuable information source to collect information on the vegetation height as an addition to the optical information from Very High Resolution (VHR) satellite imagery $(<=2 \mathrm{~m}$ resolution).

\section{Study Area and Materials}

The BIO_SOS study area, named Ederheide and Ginkelse heide, is located within the Dutch Natura 2000 site "Veluwe" in the Province of Gelderland. The Veluwe is the largest terrestrial Natura 2000 site in the Netherlands, covering approximately 91,200 ha. The Ederheide and Ginkelse heide spreads over an area of approximately 1000 ha, where heathland is the dominant land cover. While the multiple return LiDAR data for the construction of the first Actual Height Model of the Netherlands for 2003 (AHN-1, Actueel Hoogtebestand Nederland, see also www.ahn.nl) still had a precision of $15 \mathrm{~cm}$ with one point measurement per $\mathrm{m}^{2}$, the acquired AHN-2 (March 2010) has a height precision of a few $\mathrm{cm}$ and a density of approximately 15 points per $\mathrm{m}^{2}$. The absolute accuracy for a single point can be guaranteed below $3 \mathrm{~cm}$. The multiple return LiDAR data used in this study is generated by the FLI-MAP 400 laser scanner, developed by Fugro Aerial Mapping (NL). FLI-MAP 400 system is carried on board of a helicopter, integrated with high-resolution photo and video camera and a precise GPS system. The laser scanner emits 250.000 laser pulses per second, scans 250 times per second, and records up to 4 partial returns per pulse. The data is then prepared as a file with $\mathrm{X}, \mathrm{Y}, \mathrm{Z}$ coordinates indicating the location of the point. The Fli-Map sensor has a Field of View (FOV) of 60 degrees. The average flight speed at which this data is captured is around $64.8 \mathrm{~km} / \mathrm{h}$, while the flight level for this particular data is around $400 \mathrm{~m}$. The wavelength of these laser pulses is in the near-infrared wavelengths. The data provided are las data files, separated by tile and flight days, with $\mathrm{X}, \mathrm{Y}, \mathrm{Z}$, Scan Angle, RGB values, Intensity values and GPS time stamps.

\section{Method for Canopy Height Model and Habitat Classification}

The Canopy Heigth Model (CHM) was derived from the LiDAR las files using the MCCLiDAR software and LAS tools. The MCC-LiDAR tool was used for the classification of the ground points. Multiscale Curvature Classification (MCC) is an iterative multiscale 
algorithm for classifying LiDAR returns that exceed positive surface curvature thresholds, resulting in all the LiDAR measurements being classified as ground or nonground. The MCC algorithm yields a solution of classified returns that support bare-earth surface interpolation at a resolution commensurate with the sampling frequency of the LiDAR survey. Classification of ground points could also have been performed with the lasground tool of the las tools suite, but MCC-lidar was selected because of the curvature capabilities. All non-ground points were assumed to be vegetation, which is mostly true for the nature areas even if some small errors could occur, like benches and signposts. The CHM is calculated in two steps, first the $\mathrm{Z}$ component of the las points is made relative to the ground so that it represents the object height. Then the las file is rasterized into a grid with the maximum $Z$ value within one grid cell of 1 by 1 meter as the object height. CHMs are crucial in our approach of habitat mapping and monitoring. Plant life forms, as described by RAUNKIAER (1934), became the basis of for habitat mapping and monitoring (BUNCE et al. 2008, 2011). The General Habitat Categories (GHC) is the dominant life form for a given mapping unit of at least $400 \mathrm{~m}^{2}$ and uses specific precedence rules. This resulted in 140 GHCs in the newly developed habitat classification system by BUNCE et al. (2011). Examples of life forms and associated GHCs are Forest Phanerophytes (FPH), which are trees higher than 5 $\mathrm{m}$, and Shrubby Chamaephytes $(\mathrm{SCH})$, which are shrubs between 5 and $30 \mathrm{~cm}$. Other examples are Caespitose Hemicroptyphytes (CHE) as grasses and Leafy Hemicroptyphytes (LHE) as forbs. Since the woody life forms on which the majority of GHCs are based have crisp height definitions they can be easily translated from the CHM. BIO_SOS has now developed new and automated methods that are beyond current state-of-the-art in Europe and use High and Very High Resolution (VHR) remote sensing data to identify and map land cover types described by the FAO Land Cover Classification System (LCCS). These are translated subsequently with contextual and semantic information to map GHC and finally Annex I habitat types. In both LCCS and GHC categories height information of the canopy is essential information. The procedures will sit within an ecological modelling framework for automated provision of habitat maps and biodiversity indicators useful for a deeper understanding of the impacts of human induced pressures.

\section{$4 \quad$ Results and Assessment}

The result obtained from LiDAR multiple return data is a canopy height model with a spatial resolution of 1 by 1 meter and vegetation height indicated in centimetres. In eCognition several Very High Resolution Worldview-2 imagery have been used considering a peak (June 2011) and a post-peak image (September 2011) in combination with the LiDAR data of March 2010. In eCognition rules were defined for all occurring LCCS land cover classes and GHC habitat classes. An example of the classification result is given is Fig. 1. The validation for the Dutch case study (based on 12 stratified random sample sites for which habitat maps were produced) showed an overall classification accuracy of $70 \%$ for the GHC habitats and $74 \%$ for the LCCS level 3 land cover classes. Regression analysis of the percentage coverage of a specific life forms of individual habitat mapping units as derived from LiDAR and measured in the field showed an R square for FPH of 0.96, for herbaceous vegetation (CHE and LHE) an R square of 0.70 , but for SCH an R square of 0.40 . 


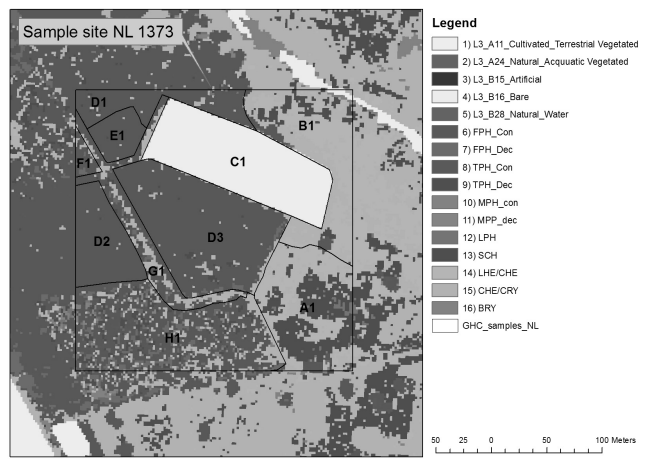

Fig. 1:

Example of a BIO_SOS EODHAM classification result in eCcognition. The first five classes indicate the LCCS level 3 classes. While all other classes belong to LCCS class A12 natural and semi-natural terrestrial vegetation, and represent the GHC habitat categories. The overlay (black lines with letter) concerns the GHC habitat map made in the field for vali dation purposes.

\section{Conclusion and Outlook}

LiDAR provides accurate height measurements for shrubs and trees, even in early spring when the objects of interest did not have any leaves. Early spring is the standard time for LiDAR measurements over the entire area of the Netherlands as the primary interest is the update of the Dutch elevation model. Regression analysis between field measurements and LiDAR measurements of the height of various plant life forms showed good results for trees but less reliable results for low shrubs as young heather for which the LiDAR pulses did not hit the ground due to its compactness (which is not the case for adult heather that has a more open structure). The BIO_SOS project is showing that the combination of LiDAR with very high resolution satellite imagery is a powerful tool for the identification of plant life forms and associated habits.

\section{References}

Bunce, R. G. H., Bogers, M. M. B., Roche, P., WalczaK, M., Geijzendorfer, I. R. \& Jongman, R. H. G (2011), Manual for Habitat and Vegetation Surveillance and Monitoring: Temperate, Mediterranean and Desert Biomes. First edition. Wageningen, Alterra report 2154.

Bunce, R. G. H., Metzger, M. J., Jongman, R. H. G., Brandt, J., De Blust, G., ElenaRossello, R., Groom, G. B., Halada, L., Hofer, G., Howard, D. C., Ková, P., Mücher, C. A., Padoa-Schioppa, E., Paelinx, D., Palo, A., Perez-Soba, M., RAMOS, I. L., Roche, P., SKÅNES, H. \& WRBKA, T. (2008), A standardized procedure for surveillance and monitoring European habitats and provision of spatial data. Landscape Ecol., 23 (1), 11-25.

GeErling, G. W., LABrAdor-Garcia, M., et al. (2007), Classification of floodplain vegetation by data fusion of spectral (CASI) and LiDAR data. International Journal of Remote Sensing, 28 (19), 4263-4284.

RAUNKIAER, C. (1934), The life forms of plants and statistical plant geography, being the collected papers of C Raunkiaer. Oxford, Clarendon.

ST-ONGE, B. (2005), LiDAR remote sensing: Overview of technology and applications. Proceedings of the 26th Canadian Symposium on Remote Sensing. 